\title{
Photoluminescence of a CVD Diamond Excited with VUV Light from a Synchrotron
}

\author{
Hsiao-Chi Lu, Yu-Chain Peng, Meng-Yeh Lin, Sheng-Lung Chou, Jen-Iu Lo, Bing-Ming Cheng \\ National Synchrotron Radiation Research Center, Hsinchu Science Park, Hsinchu, Chinese Taipei \\ Email: hopelu@nsrrc.org.tw, bmcheng@nsrrc.org.tw
}

Received August 2, 2013; revised September 5, 2013; accepted September 28, 2013

Copyright (C) 2013 Hsiao-Chi Lu et al. This is an open access article distributed under the Creative Commons Attribution License, which permits unrestricted use, distribution, and reproduction in any medium, provided the original work is properly cited.

\begin{abstract}
At the vacuum-ultraviolet (VUV) beam line of a synchrotron, an end station for photoluminescence (PL) coupled to a system to detect absorption is used to investigate the luminescence and absorption of materials. We analyzed a CVD diamond window in wavelength range 160 - $250 \mathrm{~nm}$ at 300 and $14 \mathrm{~K}$. The PL excited with VUV light enabled an identification of nitrogen defects in diamond samples. The VUV PL technique is applicable to explore advanced materials including materials with similar wide band gaps, such as boron nitride and aluminum nitride.
\end{abstract}

Keywords: Diamond; Nitrogen Defect; Photoluminescence; Synchrotron; Vacuum-Ultraviolet

\section{Introduction}

Photoluminescence (PL) arises when a sample is excited with radiation and responds by relaxation with emission of light. As PL, the material might emit light no matter what its form, shape, size or state. A great advantage of this analytical technique is the lack of necessity of further sample preparation for materials in various forms. Photoluminescence is hence well developed; numerous applications are employed for diverse materials - biomolecules, polymers, ice aggregates and polycyclic aromatic hydrocarbons.

Typical sources of excitation of PL, comprising mercury lamps, xenon lamps, tungsten filaments, tungstenhalogen lamps and various lasers, thus operate in the visible and UV ranges. For these conventional and laser sources, to tune the energy to the vacuum ultraviolet (VUV) region is difficult. For some advanced materials, especially phosphors applied in a plasma-display panel (PDP), their excitation requires light with photon energy into the VUV region. Synchrotron radiation (SR) provides an intense and continuous source of VUV light, which serves effectively as excitation to produce photoluminescence in the VUV region [1].

For this purpose, we have established an end station, coupled to a beam line of a synchrotron for photoluminescence, to investigate the luminescence of phosphors [2]. To explore new materials, we continually improve the performance of this end station. Here, we present the results of our investigation of a CVD diamond.
To prepare a CVD diamond, the most general methods involve deposition of a diamond film with plasma discharges, heated filaments and combustion. With improved CVD techniques yielding excellent and controlled rates of growth of diamond, CVD diamonds of great quality can be produced for some industrial and optical applications, but these diamond samples might contain impurities and crystal defects resulting from their growth through the gaseous phase [3]. We compared the results from IR absorption, UV absorption and VUV PL for sensitive analysis of CVD diamonds.

\section{Experiments}

Figure 1 shows an experimental setup. The VUV photoluminescence and absorption spectra are recorded at beam line 03A in Taiwan's National Synchrotron Radiation Research Center (NSSRC). In summary, for excitation, VUV light in an intense beam from this line attached to the $1.5-\mathrm{GeV}$ storage ring was dispersed with a cylindrical grating monochromator (CGM, $6 \mathrm{~m})$. The intensity of the VUV light is monitored with a gold mesh transmitting about $90 \%$ and recorded with an electrometer (Keithley 6512).

For measurement of PL, a monochromator (JobinYvon HR320) equipped with a diffraction grating (1200 lines $/ \mathrm{mm}$ ) and a photomultiplier tube (PMT, Hamamatsu R943-02) serve to record the PL spectra. To record the excitation (PLE) spectra, the dispersed emission was monitored at a selected wavelength, for which purpose 


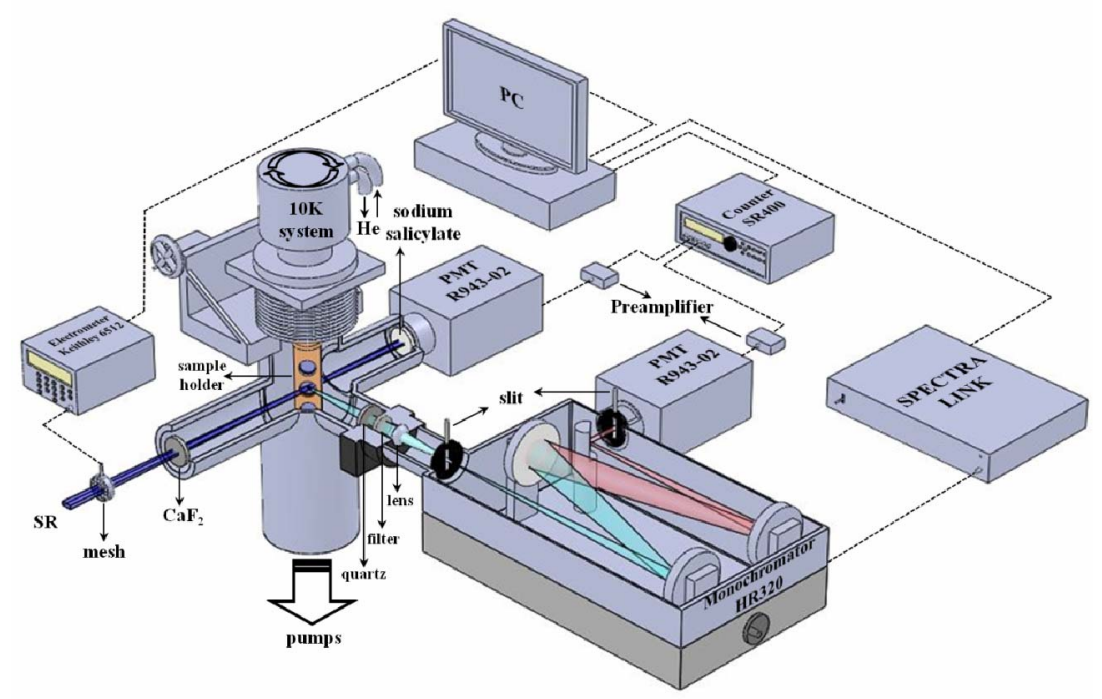

Figure 1. Experiment of photoabsorption and photoluminescence system excited with synchrotron VUV light.

the CGM beam was scanned with a grating (450 lines/ $\mathrm{mm})$. All PLE spectra were normalized with the spectral response curve of the CGM beam line. To measure the spectra of samples at a temperature below $100 \mathrm{~K}$, we attached the sample holder to the cold head of a helium closed-cycle cryostat (APD HC-DE204S); this holder was mounted on a flange such that the sample was rotatable to about $45^{\circ}$ with respect to both the incident VUV source and the entrance slit of the monochromator. The temperature of the cold head in the cryostat was controlled to better than $\pm 1 \mathrm{~K}$ during the period of data collection.

For the absorption measurements, the VUV light from the synchrotron was perpendicularly intersected to the CVD diamond samples; afterwards, the transmitted light irradiated on a glass window coated with sodium salicylate. Subsequently, the converted visible light was measured with a photomultiplier tube (Hamamatsu R943-02) in a photon-counting mode.

IR absorption spectra were recorded with a spectrometer (Bomem DA8 FTIR or Nicolet Magna 860 FTIR) attached to the infrared beamline; a detector, $\mathrm{HgCdTe}$ or DTGS, spanned the mid-IR range, $500-4000 \mathrm{~cm}^{-1}$ or $400-4000 \mathrm{~cm}^{-1}$, respectively. The IR spectra were typically measured with resolution 0.5 to $1 \mathrm{~cm}^{-1}$ and 512 to 1000 scans.

CVD diamond discs (type IIa optical grade, diameter $15 \mathrm{~mm}$ ) were purchased with thickness $1.2 \pm 0.05 \mathrm{~mm}$ (Diamond Materials Advanced Diamond Technology Company) or $1.0 \mathrm{~mm}$ (Drukker International Asia Pacific Company).

\section{Results and Discussion}

Diamonds are rarely pure and might be contaminated with hydrogen, boron, nitrogen and other elements. These impurities in diamonds are distinguishable according to their infrared spectra, which have hence served to characterize and to classify diamond samples [4]. Figure 2 displays a typical absorption spectrum of a CVD diamond disc. Intense lines, in multiplets at 1976 , 2030, 2094, 2158, 2177, 2442 and $2515 \mathrm{~cm}^{-1}$ and attributed to two-phonon absorption, are intrinsic to all diamonds $[5,6]$. For an ideal diamond, one-phonon transitions about $1330 \mathrm{~cm}^{-1}$ are forbidden according to the symmetry of a perfect lattice; the intrinsic absorption of pure diamonds is observed only in two and three-phonon regions.

Apart from these intrinsic signals, some one-phonon absorption lines due to lattice defects and impurities are discernible in impure diamonds. As examples, the presence of two adjacent substitutional nitrogen atoms (N2) in a diamond, called an A center, might produce an absorption at $1282 \mathrm{~cm}^{-1}$, whereas a line at $1177 \mathrm{~cm}^{-1}$ characterizes a B center due to four nitrogen atoms (N4) on substitutional sites symmetrically surrounding a vacancy [5]. Figure 2 contains no line attributable to an impurity or defect; this CVD diamond disc sample is, accordingly, apparently free of structural defect and chemical impurity. There is no evidence of lines attributable to nitrogen in the one-phonon region induced by defects; this diamond is then denoted as type IIa.

Figure 3 shows absorption spectra of this CVD diamond disc in wavelength region $222-250 \mathrm{~nm}$ at 300 and $14 \mathrm{~K}$. These UV absorption spectra of a diamond disc exhibit no sharp line and are sensitive to temperature. The thresholds of intense absorption of this diamond disc are recorded at $226.3 \mathrm{~nm}$ at $300 \mathrm{~K}$ and $225.8 \mathrm{~nm}$ at $14 \mathrm{~K}$; these wavelengths correspond to energies 5.479 and $5.491 \mathrm{eV}$, respectively. These absorption edges might be 
associated with indirect intrinsic electronic transitions of diamond from the valence-band maximum to the conduction-band maximum. This UV absorption threshold thus shifts $0.5 \mathrm{~nm}$ to the blue from 300 to $14 \mathrm{~K}$. The UV absorption tail also shows a pronounced temperature effect-a long tail at $300 \mathrm{~K}$ that vanishes at $14 \mathrm{~K}$. This tail is due to crystal phonons, which are frozen at low temperature. According to these UV absorption spectra, this diamond disc possesses an optical quality sufficient for it to be denoted as a window of optical grade [7].

Photoluminescence is a sensitive and powerful technique to characterize optically the impurities, defects and absorption edges of natural and synthetic diamonds. Both emission and excitation spectra of various diamond samples have been measured under diverse conditions in IR, visible, UV and X-ray regions. According to the UV absorption spectra, light suitable for use for PL excitation should have a wavelength less than $226 \mathrm{~nm}$ for this diamond window of optical grade.

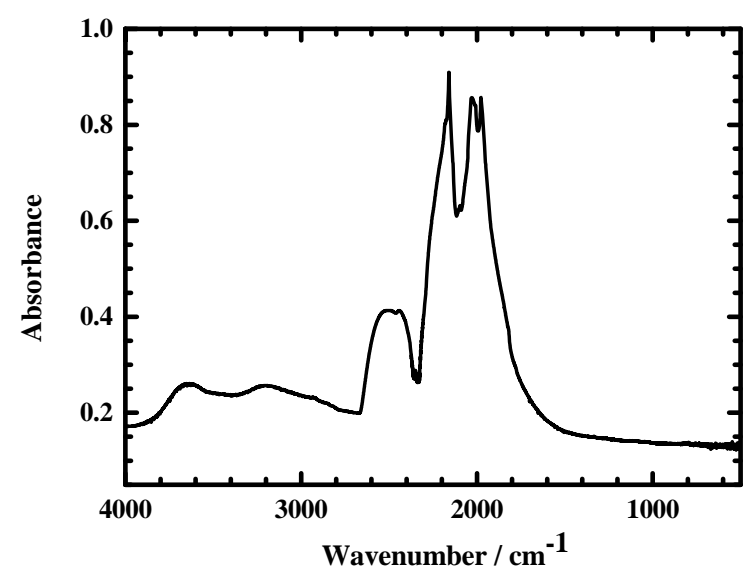

Figure 2. IR absorption spectrum of a CVD diamond disc (thickness $1.2 \pm 0.05 \mathrm{~mm}$ ).

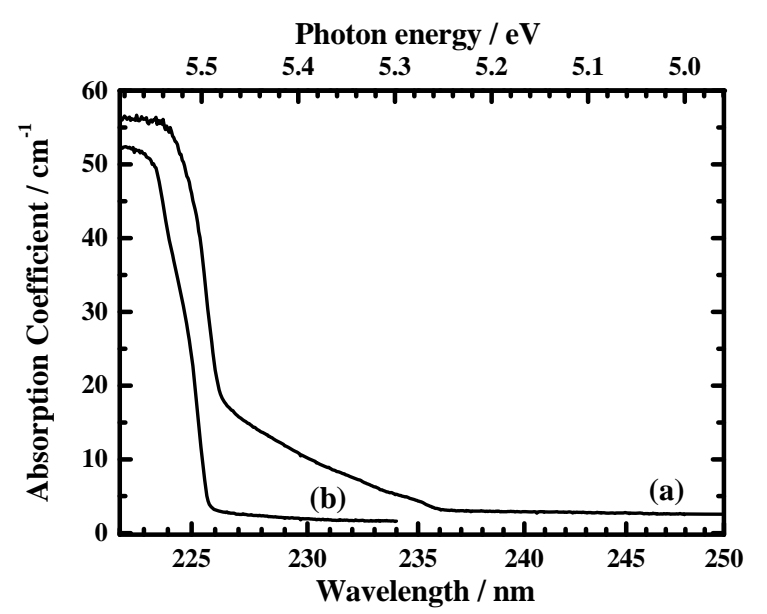

Figure 3. Absorption spectra (resolution $0.1 \mathrm{~nm}$, scan step $0.05 \mathrm{~nm}$ ) of a CVD diamond disc (thickness $1.2 \pm 0.05 \mathrm{~mm}$ ) at (a) $300 \mathrm{~K}$ and (b) $14 \mathrm{~K}$.
Upon excitation at $223 \mathrm{~nm}$ of this CVD diamond window at 300 and $14 \mathrm{~K}$, the emission spectra are displayed in Figures 4(a) and (b), respectively. Although the emission signal, as shown for $300 \mathrm{~K}$ in Figure 4(a), is weak, the broad spectrum is seen to contain two broad lines in the purple and blue regions, with maxima about 428 and $470 \mathrm{~nm}$, corresponding to photon energies 2.90 and 2.64 $\mathrm{eV}$, respectively. The PL emission spectrum accordingly reveals emission from $\mathrm{A}(\mathrm{N} 2)$ and $\mathrm{B}(\mathrm{N} 4)$ centers [5].

For the sample at $14 \mathrm{~K}$, the maximum intensity of the $\mathrm{PL}$ emission is 12 times that of the same sample at $300 \mathrm{~K}$; prominent features appear along the continuous curve. The characteristic emission feature recorded at $416.5 \mathrm{~nm}$ in Figure 4(b), is accordingly identified unambiguously as the zero-phonon line (ZPL) of the N3 center, which was confirmed to be near $416 \mathrm{~nm}$ for various diamonds [8]. Another and narrow emission line, unassigned, is observed at $501 \mathrm{~nm}$.

The photoluminescence excitation (PLE) spectra of a CVD diamond disc monitored with emission about 428 $\mathrm{nm}$ at $300 \mathrm{~K}$ and $417 \mathrm{~nm}$ at $14 \mathrm{~K}$ are presented in Figure 5; the maximum PLE intensity detected at $14 \mathrm{~K}$ is nine times that at $300 \mathrm{~K}$. The signals recorded at $300 \mathrm{~K}$ were small; no distinct feature was identified. Two PLE thresholds were derived at $235.8 \mathrm{~nm}$ (photon energy $5.258 \mathrm{eV})$ and $226.3 \mathrm{~nm}(5.479 \mathrm{eV})$. Comparison with the UV absorption spectrum in Figure 3(a) indicates that PLE and absorption possess similar optical behavior in the threshold region $223-240 \mathrm{~nm}$.

When the sample was cooled to $14 \mathrm{~K}$, PLE features appeared between $224(5.53 \mathrm{eV})$ and $180 \mathrm{~nm}(6.80 \mathrm{eV})$, as shown in Figure 5(b); PLE lines were recorded at $223.2 \mathrm{~nm}(5.555 \mathrm{eV}), 216.8 \mathrm{~nm}(5.719 \mathrm{eV}), 210.6 \mathrm{~nm}$ $(5.887 \mathrm{eV}), 205.0 \mathrm{~nm}(6.048 \mathrm{eV})$ and $200.0 \mathrm{~nm}(6.199$ $\mathrm{eV})$. According to our previous work, these PLE bands

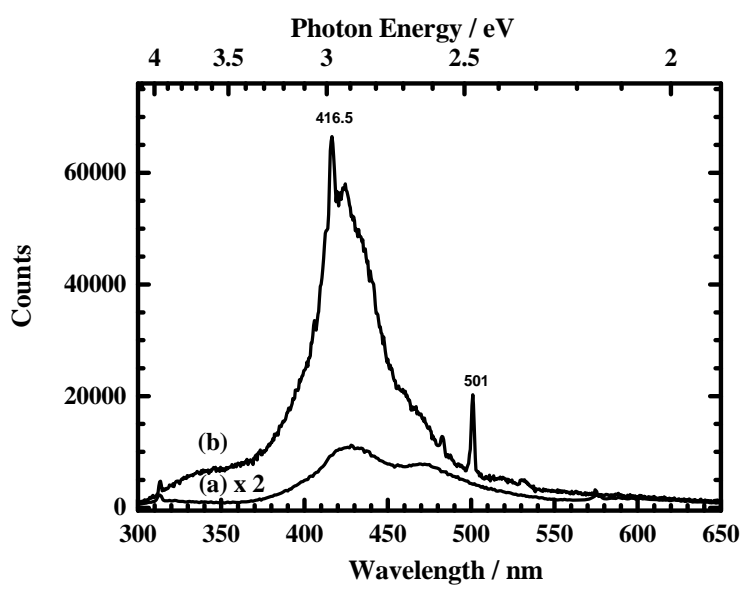

Figure 4. Photoluminescence emission spectra (resolution $0.5 \mathrm{~nm}$, scan step $0.5 \mathrm{~nm}$ ) of a CVD diamond window (thickness $1.2 \pm 0.05 \mathrm{~mm}$ ) (a) at $300 \mathrm{~K}$ excited at $223.6 \mathrm{~nm}$ and (b) at $14 \mathrm{~K}$ excited at $223.2 \mathrm{~nm}$. 


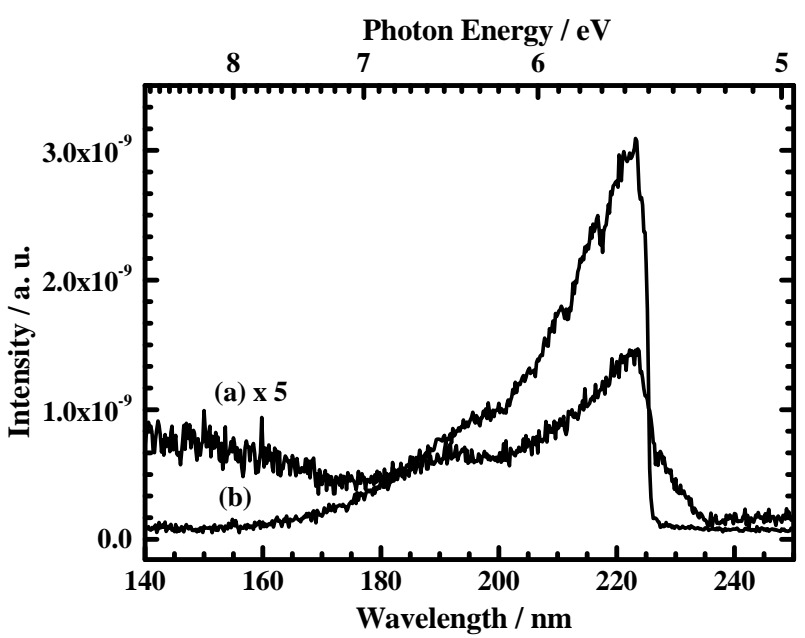

Figure 5. Photoluminescence excitation (PLE) spectra (resolution $0.2 \mathrm{~nm}$, scan step $0.2 \mathrm{~nm}$ ) of a CVD diamond window (thickness $1.2 \pm 0.05 \mathrm{~mm}$ ) (a) at $300 \mathrm{~K}$ monitored at $428.0 \mathrm{~nm}$, and (b) at $14 \mathrm{~K}$ monitored at $416.5 \mathrm{~nm}$.

are labeled as lines $\mathrm{A}(0)$ - $\mathrm{A}(4)$, respectively, of progression A of the N2 center [8]. The PLE threshold at 225.8 $\mathrm{nm}(5.491 \mathrm{eV})$ at $14 \mathrm{~K}$ approaches the UV absorption threshold displayed in Figure 3(b).

\section{Conclusion}

In summary of our results, to identify impurities and defects in a CVD diamond window of optical quality, we applied three spectrometric methods. Hence, we found that IR and UV absorption techniques are insufficiently sensitive to monitor minute impurities and defects in diamonds, whereas the VUV PL technique provides an analytical ability for nitrogen defects. We contend that the PL technique excitation with VUV light from a synchrotron can provide unique spectra and extend the capability to explore advanced materials including those with similar wide band gaps, such as boron nitride and aluminum nitride.

\section{Acknowledgements}

National Science Council of the Republic of China (grant NSC102-2113-M-213-005-MY3) and National Synchrotron Radiation Research Center provided support.

\section{REFERENCES}

[1] L.-J. Lai, H.-C. Lu, H.-K. Chen, B.-M. Cheng, M.-I Lin and T.-C. Chu, "Photoluminescence of Zirconia Films with VUV Excitation," Journal of Electron Spectroscopy and Related Phenomena, Vol. 144-147, 2005, pp. 865868. http://dx.doi.org/10.1016/j.elspec.2005.01.222

[2] H.-C. Lu, H.-K. Chen, T.-Y. Tseng, W.-L. Kuo, M. S. Alam and B.-M. Cheng, "Photoluminescence of Phosphors for PDP with VUV Excitation," Journal of Electron Spectroscopy and Related Phenomena, Vol. 144-147, 2005, pp. 983-985.

http://dx.doi.org/10.1016/j.elspec.2005.01.232

[3] A. Tallaire, A. T. Collins, D. Charles, J. Achard, R. Sussmann, A. Gicquel, M. E. Newton, A. M. Edmonds and R. J. Cruddace, "Characterisation of High-Quality Thick Single-Crystal Diamond Grown by CVD with a Low Nitrogen Addition," Diamond \& Related Materials, Vol. 15, No. 10, 2006, pp. 1700-1707.

http://dx.doi.org/10.1016/j.diamond.2006.02.005

[4] A. M. Zaitsev, "Optical Properties of Diamond: A Data Handbook," Springer-Verlag, Berlin, Heidelberg, 2001. http://dx.doi.org/10.1007/978-3-662-04548-0

[5] H.-C. Lu and B.-M. Cheng, "Analysis of Nitrogen Defects in Diamond with VUV Photoluminescence," Analytical Chemistry, Vol. 83, No. 17, 2011, pp. 6539-6544. http://dx.doi.org/10.1021/ac200808n

[6] B. Rondeau, E. Fritsch, M. Guiraud, J.-P. Chalain and F. Notari, "Three Historical 'Asteriated' Hydrogen-Rich Diamonds: Growth History and Sector-Dependent Impurity Incorporation," Diamond \& Related Materials, Vol. 13, No. 9, 2004, pp. 1658-1673.

http://dx.doi.org/10.1016/j.diamond.2004.02.002

[7] L. H. Robins, P. J. H. Tjossem, K. C. Smyth, P. Y. Barnes, E. N. Farabaugh and A. Feldman, "Photoluminescence Excitation by Bandgap Optical Absorption in Chemical Vapor Deposition Diamond Films," Journal of Applied Physics, Vol. 69, No. 6, 1991, pp. 3702-3708. http://dx.doi.org/10.1063/1.348463

[8] H.-C. Lu, M.-Y. Lin, S.-L. Chou, Y.-C. Peng, J.-I. Lo and B.-M. Cheng, "Identification of Nitrogen Defects in Diamond with Photoluminescence Excited in the 160-240 nm Region," Analytical. Chemistry, Vol. 84, No. 21, 2014, pp. 9596-9600. 